\title{
Size-dependent electron-hole exchange interaction in Si nanocrystals
}

\author{
M. L. Brongersma, ${ }^{\text {a) }}$ P. G. Kik, and A. Polman \\ FOM Institute for Atomic and Molecular Physics, Kruislaan 407, 1098 SJ Amsterdam, The Netherlands
}

\begin{abstract}
K. S. Min and Harry A. Atwater
Thomas J. Watson Laboratory of Applied Physics, California Institute of Technology, Pasadena, California 91125
\end{abstract}

(Received 4 February 1999; accepted for publication 15 November 1999)

\begin{abstract}
Silicon nanocrystals with diameters ranging from $\approx 2$ to $5.5 \mathrm{~nm}$ were formed by $\mathrm{Si}$ ion implantation into $\mathrm{SiO}_{2}$ followed by annealing. After passivation with deuterium, the photoluminescence (PL) spectrum at $12 \mathrm{~K}$ peaks at $1.60 \mathrm{eV}$ and has a full width at half maximum of $0.28 \mathrm{eV}$. The emission is attributed to the recombination of quantum-confined excitons in the nanocrystals. The temperature dependence of the PL intensity and decay rate at several energies between 1.4 and $1.9 \mathrm{eV}$ was determined between 12 and $300 \mathrm{~K}$. The temperature dependence of the radiative decay rate was determined, and is in good agreement with a model that takes into account the energy splitting between the excitonic singlet and triplet levels due to the electron-hole exchange interaction. The exchange energy splitting increases from $8.4 \mathrm{meV}$ for large nanocrystals $(\approx 5.5 \mathrm{~nm})$ to $16.5 \mathrm{meV}$ for small nanocrystals $(\approx 2 \mathrm{~nm})$. For all nanocrystal sizes, the radiative rate from the singlet state is 300-800 times larger than the radiative rate from the triplet state. (C) 2000 American Institute of Physics. [S0003-6951(00)04402-8]
\end{abstract}

Si nanostructures have received intense study, which is stimulated by their potential for use in Si-based optoelectronic devices. ${ }^{1,2}$ The photoluminescence (PL) from Si nanostructures that are well passivated by $\mathrm{H}$ or $\mathrm{SiO}_{2}$ is attributed to the recombination of quantum-confined excitons. ${ }^{3-6}$ The nanocrystals show a higher quantum efficiency for optical emission than bulk $\mathrm{Si}^{4}{ }^{4}$ and exhibit a band gap (emission energy) that can be continuously tuned over a large part of the visible spectrum and to the near infrared, by varying the size. $^{7}$ One of the most controlled methods to fabricate $\mathrm{Si}$ nanocrystals in $\mathrm{SiO}_{2}$ is ion beam synthesis. ${ }^{8-10}$ Si nanocrystals formed by this method provide an ideal system for the study of their size-dependent optical properties since the nanocrystals (1) generally have a wide size distribution, (2) are all close to spherical in shape, and (3) are well passivated by the $\mathrm{SiO}_{2}$ matrix. Si nanocrystal-doped $\mathrm{SiO}_{2}$ films made by ion beam synthesis show PL that can be attributed to two distinct sources. ${ }^{8}$ One luminescence feature is due to ion irradiation-induced defects and can be fully quenched by introducing $\mathrm{H}$ or $\mathrm{D}$ into the film. The other is attributed to the recombination of quantum-confined excitons.

In this letter, we present temperature-dependent measurements of the PL intensity and PL decay rate of ion beam synthesized Si nanocrystals in the size range of $\approx 2-5.5 \mathrm{~nm}$. The temperature dependence of the radiative decay rate of $\mathrm{Si}$ nanocrystals is determined and compared to a model introduced by Calcott et al., ${ }^{6}$ that takes into account the exchange interaction splitting of the singlet and triplet exciton energy levels. ${ }^{3,6}$ The exchange splitting energy is determined as a function of emission energy in the spectral range $1.4-1.9 \mathrm{eV}$, and compared to data for porous $\mathrm{Si}$ for energies $>1.8 \mathrm{eV}$. The ratio of the singlet and triplet radiative decay rates is determined for the first time.

${ }^{a)}$ Electronic mail: mbrong@caltech.edu
A $100 \mathrm{~nm}$ thick $\mathrm{SiO}_{2}$ film grown by wet thermal oxidation of a lightly B-doped $\mathrm{Si}(100)$ wafer was implanted at room temperature with $50 \mathrm{keV} \mathrm{Si}$ ions at a fluence of $5 \times 10^{16} \mathrm{Si} / \mathrm{cm}^{2}$. The sample was subsequently annealed at $1100{ }^{\circ} \mathrm{C}$ for $10 \mathrm{~min}$ in vacuum to induce nucleation and growth of Si nanocrystals. The presence of close to spherical Si nanocrystals was confirmed by transmission electron microscopy. Finally, the $\mathrm{SiO}_{2}$ film was implanted with 3.3 $\times 10^{15} \mathrm{D} / \mathrm{cm}^{2}$ at an energy of $600 \mathrm{eV}$ to quench the defect luminescence. ${ }^{8}$ PL spectra were taken using excitation with the $459 \mathrm{~nm}$ line of an Ar-ion laser at a power density of $\sim 1$ $\mathrm{mW} / \mathrm{mm}^{2}$. Lock-in detection was performed at a frequency of $16 \mathrm{~Hz}$. The luminescence was detected with an InGaAs photomultiplier and a grating spectrometer. The sample temperature was controlled between 12 and $300 \mathrm{~K}$ in a closedcycle He cryostat. All spectra were corrected for the system response. PL decay measurements were made after pumping to steady state with a power density of $\sim 0.2 \mathrm{~mW} / \mathrm{mm}^{2}$. The time resolution of the system was $400 \mathrm{~ns}$.

Figure 1 shows PL spectra taken at 12, 100, and $300 \mathrm{~K}$. The spectrum at $12 \mathrm{~K}$ is broad (full width at half maximum $0.28 \mathrm{eV}$ ) and peaks around $1.6 \mathrm{eV}$. When the temperature is raised, the peak intensity increases with temperature up to $100 \mathrm{~K}$ and decreases again for higher temperatures. A shift of the peak position to lower energies is observed when the temperature is increased to $300 \mathrm{~K}$, while the overall spectral shape hardly changes.

The spectrum is broad due to the wide distribution of nanocrystal sizes, each emitting at their characteristic, sizedependent energy. The full spectral range from 1.4 to $2.0 \mathrm{eV}$ corresponds to a size range of about $5.5-2 \mathrm{~nm} .{ }^{11}$ The inset in Fig. 1 shows the dependence of the peak position on temperature as obtained from PL spectra taken at various temperatures in the range from 12 to $300 \mathrm{~K}$. The peak energy decreases monotonically from 1.60 to $1.54 \mathrm{eV}$ as the tem- 


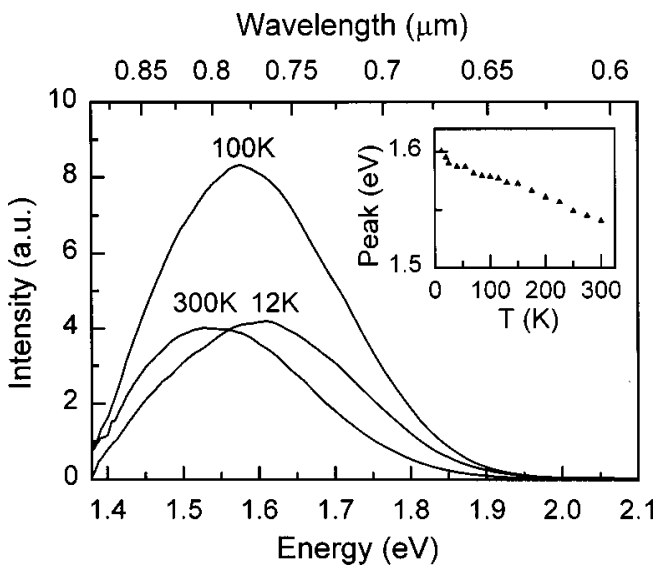

FIG. 1. Photoluminescence spectra taken at 12,100 , and $300 \mathrm{~K}$ of a $100 \mathrm{~nm}$ thick $\mathrm{SiO}_{2}$ film that contains ion beam synthesized $\mathrm{Si}$ nanocrystals with a broad size distribution. The inset shows the peak energy of these and other spectra (not shown) taken at various temperatures. Excitation at $458 \mathrm{~nm}$ $(2.71 \mathrm{eV})$ and a power density of $\approx 1 \mathrm{~mW} / \mathrm{mm}^{2}$.

perature is increased from 12 to $300 \mathrm{~K}$. The decrease can mainly be attributed to the decrease in the band gap energy with temperature. For comparison: the band gap variation with temperature for bulk $\mathrm{Si}$ is $50 \mathrm{meV}$ over the temperature range from 10 to $300 \mathrm{~K}$.

Figure 2(a) shows the dependence of the integrated PL intensity on temperature. It first increases by a factor of 2 from 12 to $100 \mathrm{~K}$ and then decreases by a similar amount when the temperature is increased to $300 \mathrm{~K}$. The fact that the PL spectrum does not change shape, but shifts with increasing temperature, suggests that the temperature dependence of the emission intensity is the same for all nanocrystal sizes that contribute to the spectrum.

PL decay traces at emission energies of 1.46, 1.55, 1.65, 1.77, and $1.90 \mathrm{eV}$ were measured at temperatures in the range from 12 to $300 \mathrm{~K}$. The decay traces are well described by a stretched exponential function,

$$
I_{\mathrm{PL}}(t)=I_{0} \exp \left[-\left(R_{\mathrm{PL}} t\right)^{\beta}\right],
$$

where $I_{0}$ is the PL intensity at $t=0, R_{\mathrm{PL}}$ an effective decay rate, and $\beta$ a constant between 0 and 1 . A typical decay trace taken at $1.65 \mathrm{eV}$ and $15 \mathrm{~K}$ is shown in the inset of Fig. 2(b). For this trace $R_{\mathrm{PL}}=6.2 \times 10^{2} \mathrm{~s}^{-1}\left(1 / R_{\mathrm{PL}}=1.6 \mathrm{~ms}\right)$ and $\beta$ $=0.65$. Figure 2 (b) shows the temperature dependence of $R_{\mathrm{PL}}$, as obtained by fitting the decay traces taken at 1.46 (dots), 1.65 (squares), and $1.90 \mathrm{eV}$ (triangles) with Eq. (1). The drawn lines serve to guide the eye. At each energy, $R_{\mathrm{PL}}$ first increases rapidly by more than an order of magnitude between 12 and $90 \mathrm{~K}$, and then slowly up to $300 \mathrm{~K}$.

The temperature dependence of $I_{\mathrm{PL}}$ and $R_{\mathrm{PL}}$ [Figs. 2(a) and 2(b)] can be described by a model ${ }^{3,6}$ that ascribes the luminescence to the recombination of strongly localized excitons in crystalline $\mathrm{Si}$ (see inset in Fig. 3). In this model, the excitonic levels are split by an energy, $\Delta$, due to the exchange interaction between the electron and hole. The lower level corresponds to a triplet state which is threefold degenerate and has a radiative decay rate $R_{T}$. The upper level corresponds to a singlet state and has a radiative decay rate $R_{S}$. This simple two-level model has been used to describe the temperature-dependent luminescence of porous silicon. There is some conflict in the literature whether this model, Downloaded 03 Apr 2006 to 131.215.225.171. Redistribution subje

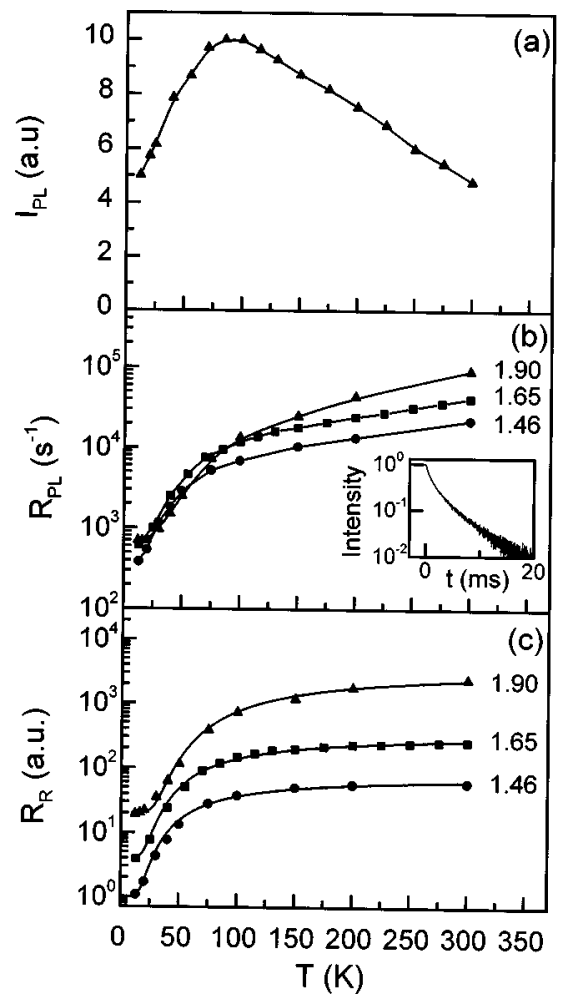

FIG. 2. (a) Temperature dependence of the integrated photoluminescence intensity, $I_{\mathrm{PL}}$, of Si nanocrystals in $\mathrm{SiO}_{2}$. (b) Temperature dependence of the photoluminescence decay rate, $R_{\mathrm{PL}}$, measured at emission energies of $1.46,1.65$, and $1.90 \mathrm{eV}$. The inset shows a typical decay trace taken at 1.65 $\mathrm{eV}$ and $15 \mathrm{~K}$. (c) Temperature dependence of the radiative rate at emission energies of $1.46,1.65$, and $1.90 \mathrm{eV}$, obtained from a multiplication of the temperature dependent $I_{\mathrm{PL}}$ data in (a) and $R_{\mathrm{PL}}$ data in (b). The solid curves are best fits of $R_{R}$ using a model that takes into account the exchange splitting of the energy levels of quantum-confined excitons in the Si nanocrystals.

that applies for nonspherical particles, would apply for spherical nanocrystals as well. ${ }^{12-14}$ In any case, the exact shape of our ion beam synthesized nanocrystals is unknown and may in fact be slightly nonspherical.

The temperature dependence of the total radiative decay rate, $R_{R}$, can be calculated by assuming thermal equilibrium between the two levels:

$$
R_{R}=\frac{3 R_{T}+R_{S} \exp (-\Delta / k T)}{3+\exp (-\Delta / k T)} .
$$

In general, the radiative decay competes with nonradiative decay channels (at a rate $R_{\mathrm{NR}}$ ) that can, for example, be provided by defects in the nanocrystal itself or at the $\mathrm{Si} / \mathrm{SiO}_{2}$ interface. At low pump power density, the decay of excited electron hole pairs is unimolecular and $R_{\mathrm{PL}}=R_{R}+R_{\mathrm{NR}}$. In low-power steady state conditions, $I_{\mathrm{PL}}$ is proportional to the quantum yield, $\eta=R_{R} /\left(R_{R}+R_{\mathrm{NR}}\right)$. Therefore, by calculating the product of the measured $I_{\mathrm{PL}}$ and $R_{\mathrm{PL}}$ at each temperature, a relative measure can be derived of the temperature dependence of the radiative decay rate $R_{R}$.

Figure 2(c) shows the calculated temperature dependence of $R_{R}$ for emission energies of $1.46,1.65$, and $1.90 \mathrm{eV}$, obtained from a multiplication of $I_{\mathrm{PL}}$ [Fig. 2(a)] and $R_{\mathrm{PL}}$ [Fig. 2(b)] at each temperature. Since these data are derived from the unnormalized $I_{\mathrm{PL}}$, each data set is expressed in arbitrary units, and has been multiplied by a different conto AIP license or copyright, see http://apl.aip.org/apl/copyright.jsp 


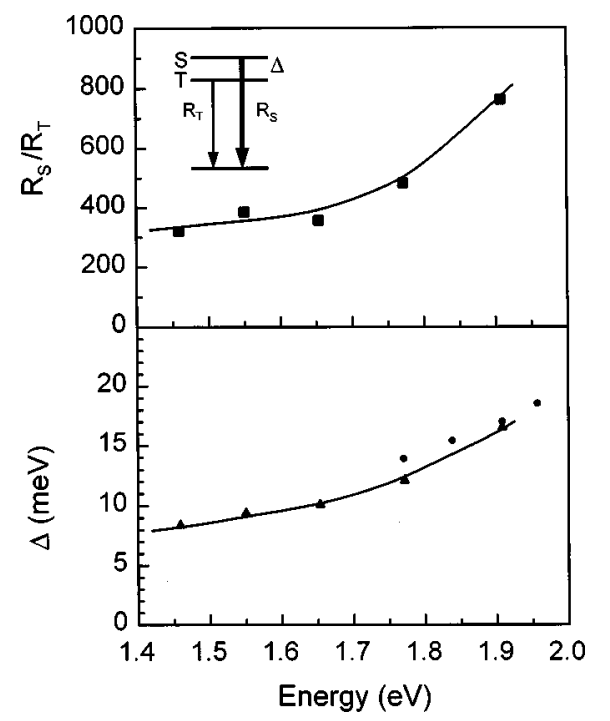

FIG. 3. (a) Ratio of the singlet $\left(R_{S}\right)$ and triplet $\left(R_{T}\right)$ radiative decay rates of quantum-confined excitons in Si nanocrystals as a function of the emission energy. The inset shows a schematic of the singlet and triplet energy levels, split by the electron-hole exchange energy $\Delta$. (b) Exchange energy as a function of the emission energy. The triangles are obtained from the measurements described in this letter. The dots represent values of $\Delta$ obtained for porous $\mathrm{Si}$ taken from Ref. 6 . The values of $R_{S} / R_{T}$ and $\Delta$ were determined by fitting Eq. (2) to the temperature dependence of the radiative rate shown in Fig. 2(c).

stant factor to facilitate comparison. The data in Fig. 2(c) can now be fitted with Eq. (2) and the drawn lines show the resulting fits. The temperature dependence of $R_{R}$ is in excellent agreement with this model. At low temperature ( $T$ $\approx 12 \mathrm{~K}$ and $k T \approx 1.0 \mathrm{meV}$ ), only the triplet state is occupied and the radiative decay rate is small. The radiative decay rate of a pure triplet state should be zero as the transition is parity forbidden. However, the spin-orbit interaction slightly mixes the singlet and triplet states, making the transition weakly allowed. At higher temperatures, the singlet state becomes populated and the radiative rate increases by more than an order of magnitude between 12 and $100 \mathrm{~K}$. When the temperature is increased further, the population in the singlet state converges to its high temperature value which is $1 / 3$ of the total triplet population, and $R_{R}$ is dominated by $R_{S}$. Indeed, $R_{R}$ shows only a small variation with temperature for $T>100 \mathrm{~K}$. The data for $1.46,1.65$, and $1.90 \mathrm{eV}$ show a slightly different behavior which can be explained by differences in the values of $R_{T}, R_{S}$, and $\Delta$, as will be shown below.

Figure 3(a) shows the ratio of $R_{S}$ to $R_{T}$ as a function of emission energy obtained by fitting Eq. (2) to the temperature dependent data of $R_{R}$ shown in Fig. 2(c) and to data taken at other energies (not shown). At all energies, $R_{S}$ is 300-800 times larger than $R_{T}$. The triangles in Fig. 3(b) show the dependence of the exchange splitting energy, $\Delta$, on the emission energy, $E$. The value of $\Delta$ increases from 8.4 to $16.5 \mathrm{meV}$ between $E=1.46$ and $1.90 \mathrm{eV}$, corresponding to a nanocrystal size range of $\approx 5.5-2 \mathrm{~nm}$. For reference, we also plot values obtained for porous $\mathrm{Si}$ for $E=1.7$ to $2.0 \mathrm{eV}^{3,6}$ These measurements cannot be directly compared to those presented in this letter, as the nanostructures in porous Si are irregularly shaped, and surrounded by an inhomogeneous dielectric, in contrast to the case for the roughly spherical nanocrystals in an $\mathrm{SiO}_{2}$ matrix in this letter. Still, the data for porous $\mathrm{Si}$ seem to follow the trend suggested by our data. This suggests that the magnitude of $\Delta$ is basically related to the emission energy. For a spherical crystallite with a diameter of $3 \mathrm{~nm}$ (emission energy $\approx 1.8 \mathrm{eV}$ ), effective mass theory predicts $\Delta=13.9 \mathrm{meV},{ }^{3}$ in good agreement with the data in Fig. 3(b). The values of $\Delta$ found here are much smaller than the $71 \mathrm{meV}$ that was reported earlier for oxidized Si nanocrystals. ${ }^{15}$ In that case, however, the luminescence was thought to originate from states at the $\mathrm{Si} / \mathrm{SiO}_{2}$ surface.

In conclusion, the temperature dependence of the photoluminescence intensity and decay rate of excitons in $\mathrm{Si}$ nanocrystals was studied. The data are in agreement with a model that takes into account the size-dependent exciton exchange splitting between the singlet and triplet states. The exchange energy splitting was found to increase from 8.4 $\mathrm{meV}$ for the large nanocrystals $(\approx 5.5 \mathrm{~nm})$ to $16.5 \mathrm{meV}$ for small nanocrystals $(\approx 2 \mathrm{~nm})$. For all nanocrystal sizes, the radiative rate from the singlet state is $300-800$ times larger than the radiative rate from the triplet state.

This work is part of the research program of FOM and was supported by NWO, the Esprit program (SCOOP) of the European Union, and the National Science Foundation.

${ }^{1}$ K. D. Hirschman, L. Tsybeskov, S. D. Duttagupta, and P. M. Fauchet, Nature (London) 384, 338 (1996).

${ }^{2}$ Y. Kanemitsu, Phys. Rep. 263, 1 (1995).

${ }^{3}$ P. D. J. Calcott, K. J. Nash, L. T. Canham, M. J. Kane, and D. Brumhead, J. Phys.: Condens. Matter 5, L91 (1993); A. G. Cullis, L. T. Canham, and P. D. J. Calcott, J. Appl. Phys. 82, 909 (1997)

${ }^{4}$ L. E. Brus, P. F. Szajowki, W. L. Wilson, T. D. Harris, S. Schuppler, and P. H. Citrin, J. Am. Chem. Soc. 117, 2915 (1995).

${ }_{5}^{5}$ S. Schuppler, S. L. Friedman, M. A. Marcus, D. L. Adler, Y. H. Xie, F. M. Ross, T. D. Harris, W. L. Brown, Y. L. Chabal, L. E. Brus, and P. H. Citrin, Phys. Rev. Lett. 72, 2648 (1994).

${ }^{6}$ P. D. J. Calcott, K. J. Nash, L. T. Canham, M. J. Kane, and D. Brumhead, J. Lumin. 57, 257 (1993).

${ }^{7}$ M. L. Brongersma, A. Polman, K. S. Min, E. Boer, T. Tambo, and H. A. Atwater, Appl. Phys. Lett. 72, 2577 (1998).

${ }^{8}$ K. S. Min, K. V. Shcheglov, C. M. Yang, H. A. Atwater, M. L. Brongersma, and A. Polman, Appl. Phys. Lett. 69, 2033 (1996).

9 J. Linnros, A. Galeckas, N. Lalic, and V. Grivickas, Thin Solid Films 297, 167 (1997).

${ }^{10}$ M. L. Brongersma, A. Polman, K. S. Min, and H. A. Atwater, J. Appl. Phys. 86, 759 (1999).

${ }^{11}$ R. T. Collins, P. M. Fauchet, and M. Tischler, Phys. Today 50, 24 (1997).

${ }^{12}$ E. Martin, C. Delerue, G. Allan, and M. Lannoo, Phys. Rev. B 50, 18258 (1994).

${ }^{13}$ A. L. Efros, M. Rosen, M. Kuno, M. Nirmal, D. J. Norris, and M. Bawendi, Phys. Rev. B 54, 4843 (1996).

${ }^{14}$ K. Leung and K. B. Whaley, Phys. Rev. B 56, 7455 (1997).

${ }^{15}$ Y. Kanemitsu, Phys. Rev. B 53, 13515 (1996). 